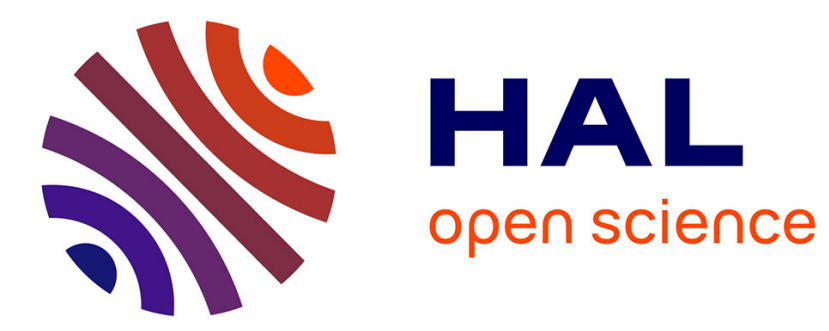

\title{
Integral Difference Ratio Functions on Integers
}

Patrick Cégielski, Serge Grigorieff, Irene Guessarian

\section{To cite this version:}

Patrick Cégielski, Serge Grigorieff, Irene Guessarian. Integral Difference Ratio Functions on Integers. Computing with new resouces, 8808, pp.210-225, 2014, Lecture Notes in Computer Science, 10.1007/978-3-319-13350-8_21. hal-01259852

\section{HAL Id: hal-01259852 \\ https://hal.science/hal-01259852}

Submitted on 25 Jan 2016

HAL is a multi-disciplinary open access archive for the deposit and dissemination of scientific research documents, whether they are published or not. The documents may come from teaching and research institutions in France or abroad, or from public or private research centers.
L'archive ouverte pluridisciplinaire HAL, est destinée au dépôt et à la diffusion de documents scientifiques de niveau recherche, publiés ou non, émanant des établissements d'enseignement et de recherche français ou étrangers, des laboratoires publics ou privés. 


\title{
Integral Difference Ratio Functions on Integers
}

\author{
Patrick Cégielski $^{1}{ }^{\star}$, Serge Grigorieff ${ }^{2}$, and Irène Guessarian ${ }^{2 \star \star \star}$ \\ 1 LACL, EA 4219, Université Paris-Est Créteil, IUT Fontainebleau-Sénart, France \\ 2 LIAFA, CNRS UMR 7089, Université Paris 7 Denis Diderot, France
}

To Jozef, on his 80th birthday, with our gratitude for sharing with us his prophetic vision of «Informatique »

\begin{abstract}
Various problems lead to the same class of functions from integers to integers: functions having integral difference ratio, i.e. verifying $f(a)-f(b) \equiv 0(\bmod (a-b))$ for all $a>b$. In this paper we characterize this class of functions from $\mathbb{Z}$ to $\mathbb{Z}$ via their $\grave{a}$ la Newton series expansions on a suitably chosen basis of polynomials (with rational coefficients). We also exhibit an example of such a function which is not polynomial but Bessel like.
\end{abstract}

Keywords: Number Theory, Lattices, Theoretical Computer Science

\section{Introduction}

We deal with the following class of functions which appears in Pin \& Silva, 2011 (see $\S 4.2$ and $\S 5.3$ in [10]), as a characterization of a special strong notion of uniform continuity.

Definition 1. Let $X \subseteq \mathbb{Z}$ (where $\mathbb{Z}$ denotes the set of integers). A map $f: X \rightarrow$ $\mathbb{Z}$ has integral difference ratio if $\frac{f(i)-f(j)}{i-j} \in \mathbb{Z}$, for all distinct $i, j \in X$.

Observe the following simple properties about these maps.

Proposition 2. 1. The set of maps $f: X \rightarrow \mathbb{Z}$ having integral difference ratio is closed under addition and multiplication. In particular, it contains all polynomials with integral coefficients.

2. The set of maps $f: X \rightarrow \mathbb{Z}$ having integral difference ratio is closed under composition.

Proof. For multiplication, use the identity $f(i) g(i)-f(j) g(j)=f(i)(g(i)-$ $g(j))+g(j)(f(i)-f(j))$.

Which non-polynomial maps have integral difference ratio? This is the question we deal with.

* Partially supported by TARMAC ANR agreement 12 BS02 00701.

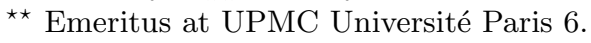


In [2] we related the integral difference ratio property to functions $f: \mathbb{N} \rightarrow \mathbb{N}$ (where $\mathbb{N}$ is the set of nonnegative integers) such that any lattice of finite subsets of $\mathbb{N}$ closed under decrement is also closed under inverse image by $f$ (Theorem 4). In $₫ 2$ we extend this result to functions $\mathbb{Z} \rightarrow \mathbb{Z}$ (Theorem 7 ).

In our paper [3] we characterized the functions $f: \mathbb{N} \rightarrow \mathbb{Z}$ having integral difference ratio in terms of their Newton expansions over the "binomial polynomials". In $\S 3$ we give a similar characterization for functions $f: \mathbb{Z} \rightarrow \mathbb{Z}$ (Theorem 16. This is the main new result of the paper; unfortunately its (very long) proof is omitted due to space limitations. Though both characterizations rely on analogous ideas, the $\mathbb{Z}$ case is not reducible to the $\mathbb{N}$ case: we have to consider $\grave{a}$ la Newton expansions over a different family of polynomials. Even though these polynomials have rational (non integer) coefficients, they map $\mathbb{Z}$ into $\mathbb{Z}$.

The characterization we give (Theorem 16 ) insures that there are uncountably many non-polynomial functions having integral difference ratio. In [3] we explicited non polynomial maps $f: \mathbb{N} \rightarrow \mathbb{Z}$ having integral difference ratio; the map $g: \mathbb{Z} \rightarrow \mathbb{Z}$ such that $g(x)=f\left(x^{2}\right)$ also has integral difference ratio and is non polynomial. In $\$ 5$ we exhibit a non-polynomial example related to Bessel functions which does not so reduce to a map $\mathbb{N} \rightarrow \mathbb{Z}$.

\section{$2 \quad$ Integral difference ratio functions and lattices}

In this section, we extend Theorem 4 of our paper [2] to functions $\mathbb{Z} \rightarrow \mathbb{Z}$.

A lattice of subsets of a set $X$ is a family of subsets of $X$ such that $L \cap M$ and $L \cup M$ are in $\mathcal{L}$ whenever $L, M \in \mathcal{L}$. Let $f: X \rightarrow X$. A lattice $\mathcal{L}$ of subsets of $X$ is closed under $f^{-1}$ if $f^{-1}(L) \in \mathcal{L}$ whenever $L \in \mathcal{L}$. Closure under decrement means closure under $S u c^{-1}$, where $S u c$ is the successor function.

We let $\mathcal{P}(X)$ denote the class of subsets of $X$. For $L \subseteq \mathbb{Z}$ and $t \in \mathbb{Z}$ we let $L-t=\{x-t \mid x \in L\}$.

Proposition 3. Let $X$ be $\mathbb{N}$ or $\mathbb{Z}$ or $\mathbb{N}_{\alpha}=\{x \in \mathbb{Z} \mid x \geq \alpha\}$ with $\alpha \in \mathbb{Z}$. For $L$ a subset of $X$ let $\mathcal{L}_{X}(L)$ be the family of sets of the form $\bigcup_{j \in J} \bigcap_{i \in I_{j}} X \cap(L-i)$ where $J$ and the $I_{j}$ 's are finite non empty subsets of $\mathbb{N}$. Then $\mathcal{L}_{X}(L)$ is the smallest sublattice of $\mathcal{P}(X)$ containing $L$ and closed under decrement.

The following characterization is proved in [2]:

Theorem 4. Let $f: \mathbb{N} \longrightarrow \mathbb{N}$ be a non decreasing function. The following conditions are equivalent:

$(1)_{\mathbb{N}}$ For every finite subset $L$ of $\mathbb{N}$, the lattice $\mathcal{L}_{\mathbb{N}}(L)$ is closed under $f^{-1}$.

$(1)_{\mathbb{N}}$ The function $f$ has integral difference ratio and $f(a) \geq a$ for all $a \in \mathbb{N}$.

$(3)_{\mathbb{N}}$ For every regular subset $L$ of $\mathbb{N}$ the lattice $\mathcal{L}_{\mathbb{N}}(L)$ is closed under $f^{-1}$.

In order to extend Theorem 4 to functions $\mathbb{Z} \rightarrow \mathbb{Z}$, we need the $\mathbb{Z}$-version of Lemma 3.1 in [2].

Lemma 5. Let $f: \mathbb{Z} \rightarrow \mathbb{Z}$ be a nondecreasing function such that $f(x)-f(y) \equiv$ $0 \bmod (x-y)$ for every $x>y \in \mathbb{Z}$. Then, for any set $L \subseteq \mathbb{Z}$, we have $f^{-1}(L)=$ $\bigcup_{a \in f^{-1}(L)} \bigcap_{t \in L-a}(L-t)$. 
Proof. Let $a \in f^{-1}(L)$. As $t \in L-a \Leftrightarrow a \in L-t$, we have $a \in \bigcap_{t \in L-a} L-t$, proving inclusion $\subseteq$.

For the other inclusion, let $b \in \bigcap_{t \in L-a} L-t$ with $a \in f^{-1}(L)$. To prove that $f(b) \in L$, we argue by way of contradiction. Suppose $f(b) \notin L$. Since $f(a) \in L$ we have $a \neq b$. The condition on $f$ insures the existence of $k \in \mathbb{Z}$ such that $f(b)-f(a)=k(b-a)$. In fact, $k \in \mathbb{N}$ since $f$ is nondecreasing.

Suppose first that $a<b$. Since $k \in \mathbb{N}$ and $f(a)+k(b-a)=f(b) \notin L$ there exists a least $r \in \mathbb{N}$ such that $f(a)+r(b-a) \notin L$. Moreover, $r \geq 1$ since $f(a) \in L$. Let $t=f(a)-a+(r-1)(b-a)$. By minimality of $r$, we get $t+a=f(a)+(r-1)(a-b) \in L$. Now $t+a \in L$ implies $t+b \in L$. But $t+b=f(a)+r(b-a) \notin L$, this contradicts the definition of $r$.

Suppose next that $a>b$. Since $k \in \mathbb{N}$ and $f(b)+k(a-b)=f(a) \in L$ there exists a least $r \in \mathbb{N}$ such that $f(b)+r(a-b) \in L$. Moreover, $r \geq 1$ since $f(b) \notin L$. Let $t=f(b)-b+(r-1)(a-b)$. By minimality of $r$, we get $t+b=f(b)+(r-1)(a-b) \notin L$. Now $t+a \in L$ implies $t+b \in L$, contradiction.

We need also recall the notions of recognizable and rational subsets: a subset $L$ of a monoid $X$ is rational if it can be generated from a finite set by unions, products and star; $L$ is recognizable if there exists a morphism $\phi: X \longrightarrow M$, with $M$ a finite monoid, and $F$ a finite subset of $M$ such that $L=\phi^{-1}(F)$. For $\mathbb{N}$, recognizable and rational subsets coincide and are called regular subsets of $\mathbb{N}$. For $\mathbb{Z}$, recognizable subsets are finite unions of arithmetic sequences, while rational subsets are unions of the form $F \cup P \cup-N$, with $F$ finite, and $P, N$ two regular subsets of $\mathbb{N}$; i.e. a recognizable subset of $\mathbb{Z}$ is also rational, but the converse is false.

Definition 6. 1. A subset $L \subseteq \mathbb{N}$ is regular if it is the union of a finite set with finitely many arithmetic progressions, i.e. $L=F \cup(R+d \mathbb{N})$ with $d \geq 1$, $F, R \subseteq\{x \mid 0 \leq x<d\}$ (possibly empty).

2. A subset $L \subseteq \mathbb{Z}$ is rational if it is of the form $L=L^{+} \cup\left(-L^{-}\right)$where $L^{+}, L^{-}$ are regular subsets of $\mathbb{N}$, i.e. $L=-(d+S+d \mathbb{N}) \cup F \cup(d+R+d \mathbb{N})$ with $d \geq 1$, $R, S \subseteq\{x \mid 0 \leq x<d\}, F \subseteq\{x \mid-d<x<d\}$ (possibly empty). See [1].

3. A subset $L \subseteq \mathbb{Z}$ is recognizable if it is of the form $L=(F+d \mathbb{Z})$ with $d \geq 1$, $F \subseteq\{x \mid 0 \leq x<d\}$

We can now extend Theorem 4 to functions $\mathbb{Z} \rightarrow \mathbb{Z}$.

Theorem 7. Let $f: \mathbb{Z} \longrightarrow \mathbb{Z}$ be a non decreasing function. The following conditions are equivalent:

$(1)_{\mathbb{Z}}$ For every finite subset $L$ of $\mathbb{Z}$, the lattice $\mathcal{L}_{\mathbb{Z}}(L)$ is closed under $f^{-1}$.

$(2)_{\mathbb{Z}}$ The function $f$ has integral difference ratio and $f(a) \geq a$ for all $a \in \mathbb{Z}$.

$(3)_{\mathbb{Z}}$ For every recognizable subset $L$ of $\mathbb{Z}$ the lattice $\mathcal{L}_{\mathbb{Z}}(L)$ is closed under $f^{-1}$.

Proof. • $(1)_{\mathbb{Z}} \Rightarrow(2)_{\mathbb{Z}}$. Assume $(1)_{\mathbb{Z}}$ holds. We first prove inequality $f(x) \geq x$ for all $x \in \mathbb{Z}$. Observe that (by Proposition 3$) \mathcal{L}_{\mathbb{Z}}(\{z\})=\left\{X \in \mathcal{P}_{<\omega}(\mathbb{Z}) \mid\right.$ $X=\emptyset$ or $\max X \leq z\}$. In particular, letting $z=f(x)$ and applying $(1)_{\mathbb{Z}}$ with $\mathcal{L}(\{f(x)\})$, we get $f^{-1}\left(\{f(x\}) \in \mathcal{L}_{\mathbb{Z}}(\{f(x)\})\right.$ hence $x \leq \max \left(f^{-1}(\{f(x\})) \leq\right.$ $f(x)$. 
To show that $f$ has integral difference ratio, we reduce to the $\mathbb{N}$ case.

For $\alpha \in \mathbb{Z}$, let $S u c_{\alpha}: \mathbb{N}_{\alpha} \rightarrow \mathbb{N}_{\alpha}$ be the successor function on $\mathbb{N}_{\alpha}=\{z \in \mathbb{Z} \mid$ $z \geq \alpha\}$. The structures $\langle\mathbb{N}, S u c\rangle$ and $\left\langle\mathbb{N}_{\alpha}, S u c_{\alpha}\right\rangle$ are isomorphic. Since $f(x) \geq x$ for all $x \in \mathbb{Z}$, the restriction $f \uparrow \mathbb{N}_{\alpha}$ maps $\mathbb{N}_{\alpha}$ into $\mathbb{N}_{\alpha}$. In particular, using Theorem 4 conditions $(1)_{\mathbb{N}_{\alpha}}$ and $(2)_{\mathbb{N}_{\alpha}}$ (relative to $f \uparrow \mathbb{N}_{\alpha}$ ) are equivalent.

We show that condition $(2)_{\mathbb{N}_{\alpha}}$ holds. Let $L \subseteq \mathbb{N}_{\alpha}$ be finite. Condition $(1)_{\mathbb{Z}}$ insures that $\mathcal{L}_{\mathbb{Z}}(L)$ is closed under $f^{-1}$. In particular, $f^{-1}(L) \in \mathcal{L}_{\mathbb{Z}}(L)$. Using Proposition 3. we get $f^{-1}(L)=\bigcup_{j \in J} \bigcap_{i \in I_{j}}(L-i)$ for finite $J, I_{j}$ 's included in $\mathbb{N}$ hence $\left(f\left\lceil\mathbb{N}_{\alpha}\right)^{-1}(L)=f^{-1}(L) \cap \mathbb{N}_{\alpha}=\bigcup_{j \in J} \bigcap_{i \in I_{j}}\left(\mathbb{N}_{\alpha} \cap(L-i)\right) \in \mathcal{L}_{\mathbb{N}_{\alpha}}(L)\right.$. This proves condition $(1)_{\mathbb{N}_{\alpha}}$. Since $(1)_{\mathbb{N}_{\alpha}} \Rightarrow(2)_{\mathbb{N}_{\alpha}}$ we see that $f\left\lceil\mathbb{N}_{\alpha}\right.$ has integral difference ratio Now, $\alpha$ is arbitrary in $\mathbb{Z}$ and the integral difference ratio property of $f \uparrow \mathbb{N}_{\alpha}$ for all $\alpha \in \mathbb{Z}$ yields the integral difference ratio property for $f$. Thus, condition $(2)_{\mathbb{Z}}$ holds.

- $(2)_{\mathbb{Z}} \Rightarrow(3)_{\mathbb{Z}}$. Assume $(2)_{\mathbb{Z}}$. It is enough to prove that $f^{-1}(L) \in \mathcal{L}_{\mathbb{Z}}(L)$ whenever $L$ is recognizable. Let $L=(F+d \mathbb{Z})$ with $d \geq 1, F=\left\{f_{1}, \cdots, f_{n}\right\} \subseteq$ $\{x \mid 0 \leq x<d\}$. Then $f$ is not constant since $f(x) \geq x$ for all $x \in \mathbb{Z}$. Also, $f^{-1}(\alpha)$ is finite for all $\alpha$ : let $b$ be such that $f(b)=\beta \neq \alpha$, by the integral difference ratio property the nonzero integer $\alpha-\beta$ is divided by $a-b$ for all $a \in f^{-1}(\alpha)$ hence $f^{-1}(\alpha)$ is finite. $f^{-1}(F)$ is thus finite too. Moreover, $L-t=F-t+d \mathbb{Z}=$ $L-t-d+d \mathbb{Z}=L-t-d=L-t+d+d \mathbb{Z}=L-t+d$, hence there are only finitely many $L-t$ 's. By Lemma 5 we have $f^{-1}(L)=\bigcup_{a \in f^{-1}(F)} \bigcap_{t \in L-a}(L-t)$; as there are only a finite number of $L-t$ 's, all union and intersections reduce to finite unions and intersections and $f^{-1}(L) \in \mathcal{L}_{\mathbb{Z}}(L)$.

- $(3)_{\mathbb{Z}} \Rightarrow(1)_{\mathbb{Z}}$. Every finite subset is recognizable hence the result.

Example 8. Theorem $4(3)_{\mathbb{N}}$ does not extend to rational subsets of $\mathbb{Z}$. Consider $L=(6+10 \mathbb{N})$ and $f(x)=x^{2} ; L$ is rational and $f$ has integral difference ratio. However $f^{-1}(L)=(\{4,6\}+10 \mathbb{N}) \cup-(\{4,6\}+10 \mathbb{N})$ does not belong to $\mathcal{L}_{\mathbb{Z}}(L)$ : $f^{-1}(L)$ contains infinitely many negative numbers, while each $L-t$ for $t \in f^{-1}(L)$ contains only finitely many negative numbers; hence any finite union of finite intersections of $L-t$ 's can contain only a finite number of negative numbers and cannot be equal to $f^{-1}(L)$.

\section{Newton series expansions of functions having integral difference ratio}

Elementary algebra shows that all polynomials have integral difference ratio. To obtain non polynomial function having integral difference ratio functions, we need a precise characterization via Newton series.

\subsection{Newton basis for functions $\mathbb{N} \rightarrow \mathbb{Z}$}

Definition 9. Let $X=\mathbb{N}$ or $X=\mathbb{Z}$. A sequence of one-variable polynomials $\left(P_{k}\right)_{k \in \mathbb{N}}$ with rational coefficients is a Newton basis for maps $X \rightarrow \mathbb{Z}$ if the following conditions are satisfied: 
(1) For every $x \in X$ and $k \in \mathbb{N}, P_{k}(x)$ is in $\mathbb{Z}$.

(2) For every $x \in X$, the set $\left\{k \in \mathbb{N} \mid P_{k}(x) \neq 0\right\}$ is finite.

(3) The correspondence which associates to a sequence $\left(a_{k}\right)_{k \in \mathbb{N}} \in \mathbb{Z}^{\mathbb{N}}$ the map $f: X \rightarrow \mathbb{Z}$ such that

$$
f(x)=\sum_{k \in \mathbb{N}} a_{k} P_{k}(x)
$$

is a bijection between sequences in $\mathbb{Z}^{\mathbb{N}}$ and maps $X \rightarrow \mathbb{Z}$.

The right side of equation (1) is called the Newton series expansion of $f$.

The following result (cf. [3]) dates back to Newton.

Proposition 10. The binomial polynomials $\left(\begin{array}{l}x \\ k\end{array}\right)=\frac{\prod_{i=0}^{k-1}(x-i)}{k !}, k \in \mathbb{N}$ (with $\left(\begin{array}{l}x \\ 0\end{array}\right)=1$ ), constitute a Newton basis for maps $\mathbb{N} \rightarrow \mathbb{Z}$.

\subsection{Characterization of functions $\mathbb{N} \rightarrow \mathbb{Z}$ having integral difference ratio}

Definition 11. For $k \in \mathbb{N}, k \geq 1, l c m(k)$ is the least common multiple of all positive integers less than or equal to $k$. By convention, $\operatorname{lcm}(0)=1$.

We proved in $[3]$ the following characterization of functions $\mathbb{N} \rightarrow \mathbb{Z}$ having integral difference ratio:

Theorem 12. Let $f: \mathbb{N} \rightarrow \mathbb{Z}$ be a function with Newton expansion $\sum_{k \in \mathbb{N}} a_{k}\left(\begin{array}{l}x \\ k\end{array}\right)$.

The following conditions are equivalent:

(1) $f$ has integral difference ratio.

(2) $\operatorname{lcm}(k)$ divides $a_{k}$ for all $k \in \mathbb{N}$.

\subsection{A Newton basis for functions $\mathbb{Z} \rightarrow \mathbb{Z}$}

The polynomials $\left(\begin{array}{l}x \\ k\end{array}\right)$ are not a Newton basis for maps $\mathbb{Z} \rightarrow \mathbb{Z}$ since condition (2) of Definition 9 fails for all negative $x$ and all $k \in \mathbb{N}$. We design another sequence of polynomials tailored for $\mathbb{Z} \rightarrow \mathbb{Z}$ maps.

Definition 13. The $\mathbb{Z}$-Newtonian polynomials are defined as follows:

$$
P_{0}(x)=1 \quad, \quad P_{2 k}(x)=\frac{1}{(2 k) !} \prod_{i=-k+1}^{i=k}(x-i) \quad, \quad P_{2 k+1}(x)=\frac{1}{(2 k+1) !} \prod_{i=-k}^{i=k}(x-i)
$$


Let us explicit the first polynomials in the above sequence:

$$
\begin{array}{r}
P_{0}(x)=1 \quad P_{1}(x)=x \quad P_{2}(x)=\frac{x(x-1)}{2 !} \quad P_{3}(x)=\frac{(x+1) x(x-1)}{3 !} \\
P_{4}(x)=\frac{(x+1) x(x-1)(x-2)}{4 !} \quad P_{5}(x)=\frac{(x+2)(x+1) x(x-1)(x-2)}{5 !} \\
P_{6}(x)=\frac{(x+2)(x+1) x(x-1)(x-2)(x-3)}{6 !} \quad \ldots
\end{array}
$$

Proposition 14. The $\mathbb{Z}$-Newtonian polynomials define maps on $\mathbb{Z}$ which take values in $\mathbb{Z}$ and satisfy the following equations for $k, n \in \mathbb{N}$,

$$
\begin{array}{cl}
P_{2 k+1}(n)=\left\{\begin{array}{cl}
\left(\begin{array}{c}
k+n \\
2 k+1
\end{array}\right) \text { if } n>k \\
0 \text { if } 0 \leq n \leq k
\end{array}\right. & P_{2 k}(n)=\left\{\begin{array}{l}
\left(\begin{array}{c}
k+n-1 \\
2 k
\end{array}\right) \text { if } n>k \\
0 \text { if } 0 \leq n \leq k
\end{array}\right. \\
P_{2 k+1}(-n)=-P_{2 k+1}(n) & P_{2 k}(-n)=\left\{\begin{array}{c}
\left(\begin{array}{c}
k+n \\
2 k
\end{array}\right) \text { if } n \geq k \\
0 \text { if } 0 \leq n<k
\end{array}\right.
\end{array}
$$

Proof. Observe that, for any $a, b, x \in \mathbb{Z}$ such that $a<0 \leq b$, we have

$$
\frac{1}{(b-a+1) !} \prod_{i=a}^{i=b}(x-i)= \begin{cases}\left(\begin{array}{c}
x-a \\
b-a+1
\end{array}\right) & \text { if } x>b \\
0 & \text { if } a \leq x \leq b \\
(-1)^{b-a+1}\left(\begin{array}{c}
|x|+b \\
b-a+1
\end{array}\right) & \text { if } x<a\end{cases}
$$

Thus, the $P_{n}$ 's map $\mathbb{Z}$ into $\mathbb{Z}$ and satisfy conditions (2) and (3).

Proposition 15. The $\mathbb{Z}-$ Newtonian polynomials are a Newton basis for maps $\mathbb{Z} \rightarrow \mathbb{Z}$.

Proof. Conditions (2), (3) in Proposition 14 insure that equation (1) of Definition 9 reduces to

$$
f(x)=\sum_{n \in\{0, \ldots, 2|x|+1\}} a_{n} P_{n}(x)
$$

which involves a finite sum. Moreover, all terms of this sum are in $\mathbb{Z}$ when the $a_{n}$ 's are in $\mathbb{Z}$. Thus, for any sequence $\left(a_{n}\right)_{n \in \mathbb{N}}$ of integers in $\mathbb{Z}$, equation (4) defines a map $f: \mathbb{Z} \rightarrow \mathbb{Z}$.

To prove the converse, observe that the instances of equation (4) can be written

$$
\begin{array}{ccrl}
f(0)=a_{0} & f(1)=a_{0}+a_{1} & f(2)=a_{0}+2 a_{1}+a_{2}+a_{3} & \ldots \\
f(-1) & =a_{0}-a_{1}+a_{2} & f(-2) & =a_{0}-2 a_{1}+3 a_{2}-a_{3}+a_{4}
\end{array} \ldots
$$


In general, for $k \geq 1$, Proposition 14 yields

$$
\begin{aligned}
f(2 k) & =L_{2 k}\left(a_{0}, \ldots, a_{4 k-2}\right)+a_{4 k-1} \quad f(2 k+1)=L_{2 k+1}\left(a_{0}, \ldots, a_{4 k}\right)+a_{4 k+1} \\
f(-2 k) & =L_{-2 k}\left(a_{0}, \ldots, a_{4 k-1}\right)+a_{4 k} \quad f(-2 k-1)=L_{-2 k-1}\left(a_{0}, \ldots, a_{4 k+1}\right)+a_{4 k+2}
\end{aligned}
$$

where $L_{n}\left(a_{0}, \ldots, a_{2 n-2}\right)$ and $L_{-n}\left(a_{0}, \ldots, a_{2 n-1}\right)$ are linear combinations of the $a_{i}$ 's with coefficients in $\mathbb{Z}$. This shows that, given any $f: \mathbb{Z} \rightarrow \mathbb{Z}$, there is a unique sequence of coefficients $\left(a_{n}\right)_{n \in \mathbb{N}}$ making equation (1) of Definition 9 true, and all these coefficients are in $\mathbb{Z}$.

\section{Functions $\mathbb{Z} \rightarrow \mathbb{Z}$ having integral difference ratio}

We can now state the main result of the paper which characterizes the functions $f: \mathbb{Z} \rightarrow \mathbb{Z}$ having integral difference ratio,

Theorem 16. Let $\sum_{k \in \mathbb{N}} a_{k} P_{k}(x)$ be the $\mathbb{Z}-N e w t o n i a n$ expansion of a function $f: \mathbb{Z} \rightarrow \mathbb{Z}$. Then the following conditions are equivalent:

(1) $f$ has integral difference ratio,

(2) $l c m(k)$ divides $a_{k}$ for all $k$.

The next two subsections are devoted to sketching the proof of Theorem 16

\subsection{Some properties involving the unary least common multiple function $l \mathrm{~cm}$ and binomial coefficients}

The unary function lcm (cf. Definition 11) has many interesting properties and recently regained interest, cf. [12 9,5 6, 4]. We state four lemmas used in the proof of Theorem 16. They link the $l \mathrm{~cm}$ function and binomial coefficients. Lemma 17 already appears in [3. Lemma 19 is a variation tailored for the $\mathbb{Z}$ case of results in 3 . Lemma 18 is a crucial specific result with a very long proof. For lack of space proofs are not included; they can be found in ArXiv.

Lemma 17. If $0 \leq n-k<p \leq n$ then $p$ divides $\operatorname{lcm}(k)\left(\begin{array}{l}n \\ k\end{array}\right)$.

Lemma 18. If $p \geq 0$ then $2(p+k)$ divides $\operatorname{lcm}(2 k)\left(\begin{array}{c}p+2 k-1 \\ 2 k-1\end{array}\right)$.

Lemma 19. If $n, k, b \in \mathbb{N}$ and $b \geq k$ then $n$ divides $A_{k, b}^{n}=\operatorname{lcm}(k)\left(\left(\begin{array}{c}b+n \\ k\end{array}\right)-\left(\begin{array}{l}b \\ k\end{array}\right)\right)$.

Lemma 20. Let $B(n, k, i)=\left(\begin{array}{c}n+k-1 \\ 2 k\end{array}\right)-\left(\begin{array}{c}i+k \\ 2 k\end{array}\right)$ and $C(n, k, i)=\left(\begin{array}{c}n+k \\ 2 k+1\end{array}\right)+$ $\left(\begin{array}{c}i+k \\ 2 k+1\end{array}\right)$.

For all $n \geq 2$, and $1 \leq i \leq n-1$, the following hold

$$
\begin{aligned}
& n+i \text { divides } \operatorname{lcm}(2 k) B(n, k, i) \quad \text { for } 1 \leq k \leq i \\
& n+i \text { divides } l c m(2 k+1) C(n, k, i) \quad \text { for } 0 \leq k \leq i
\end{aligned}
$$




\subsection{Proof of implication $(1) \Rightarrow(2)$ in Theorem 16}

In this subsection we assume that $f: \mathbb{Z} \rightarrow \mathbb{Z}$ has integral difference ratio and that $f(x)=\sum_{k \in \mathbb{N}} a_{k} P_{k}(x)$ is its $\mathbb{Z}$-Newtonian expansion. To prove that $l c m(n)$ divides $a_{n}$ we have to prove that $i$ divides $a_{n}$ for all $i \leq n$. To give the flavor of the proof, we look at the first values of $n$. We have:

$$
\begin{array}{ll}
f(0)=a_{0} & f(-1)=a_{0}-a_{1}+a_{2}, \\
f(1)=a_{0}+a_{1} & f(-2)=a_{0}-2 a_{1}+3 a_{2}-a_{3}+a_{4} \\
f(2)=a_{0}+2 a_{1}+a_{2}+a_{3} & f(-3)=a_{0}-3 a_{1}+6 a_{2}-4 a_{3}+5 a_{4}-a_{5}+a_{6} \\
f(3)=a_{0}+3 a_{1}+3 a_{2}+4 a_{3}+a_{4}+a_{5} & f\left(a_{1}\right)
\end{array}
$$

Applying the integral difference ratio property, we see that

$$
\begin{array}{llrl}
2 \text { divides } & f(1)-f(-1)=2 a_{1}-a_{2} & \text { hence } 2 \text { divides } a_{2} \\
2 \text { divides } & f(2)-f(0)=2 a_{1}+a_{2}+a_{3} & \text { hence 2 divides } a_{3} \\
3 \text { divides } & f(2)-f(-1)=3 a_{1}+a_{3} & \text { hence 3 divides } a_{3} \\
2 \text { divides } & f(-2)-f(0)=-2 a_{1}+3 a_{2}-a_{3}+a_{4} & \text { hence 2 divides } a_{4} \\
3 \text { divides } & f(-2)-f(1)=-3 a_{1}+3 a_{2}-a_{3}+a_{4} & & \text { hence 3 divides } a_{4} \\
4 \text { divides } & f(2)-f(-1)=4 a_{1}-2 a_{2}+2 a_{3}-a_{4} & & \text { hence } 4 \text { divides } a_{4}
\end{array}
$$

By induction on $n \geq 1$, we prove the property

$\mathcal{I}(n): \quad \operatorname{lcm}(2 n-1)$ divides $a_{2 n-1}$ and $\operatorname{lcm}(2 n)$ divides $a_{2 n}$.

The cases $n=1,2$ have just been done. The inductive step is split in four cases. Assuming $\mathcal{I}(j)$ for all $j<n$, we can prove that (i), (ii), (iii) and (iv) below hold

(i) Middle number $n \quad n$ divides $a_{2 n-1}$ and $n$ divides $a_{2 n}$

(ii) Below the middle If $2 \leq i<n$ then $i$ divides $a_{2 n-1}$ and $a_{2 n}$.

(iii) Above the middle, case $a_{2 n-1}$ If $1 \leq i \leq n-1$ then $n+i$ divides $a_{2 n-1}$

(iv) Above the middle, case $a_{2 n}$ If $1 \leq i \leq n$ then $n+i$ divides $a_{2 n}$

(i) to (iv), together with the base cases complete the proof of Theorem 16 $(1) \Rightarrow(2)$.

\subsection{Proof of implication $(2) \Rightarrow(1)$ in Theorem 16}

We assume that the $\mathbb{Z}$-Newton expansion $\sum_{n \in \mathbb{N}} a_{k} P_{k}(x)$ of $f: \mathbb{Z} \rightarrow \mathbb{Z}$ is such that $l c m(n)$ divides $a_{n}$ for all $n$. We want to prove that $f$ has integral difference ratio. As for given $i, j \in \mathbb{Z}, f(i)-f(j)$ is a sum of finitely many $a_{n} P_{n}(i)-a_{n} P_{n}(j)$, it suffices to prove that each function $x \mapsto \operatorname{lcm}(n) P_{n}(x)$ has integral difference ratio. Let $j<i, i, j \in \mathbb{Z}$. To prove that $i-j$ divides $l c m(n)\left(P_{n}(i)-P_{n}(j)\right)$, we argue by disjunction of cases on the parity of $n$ and the signs of $i, j$, i.e. relative to the positions of $i, j$ with respect to the intervals $]-\infty,-k],[-k, k],[k,+\infty[$ for $k=\lfloor n / 2\rfloor$. We rely on conditions 2, 3 in Proposition 14

1. Case $n=2 k$ and $i, j \in]-\infty,-k]$. Then $P_{2 k}(i)-P_{2 k}(j)=\left(\begin{array}{c}k+|i| \\ 2 k\end{array}\right)-$ $\left(\begin{array}{c}k+|j| \\ 2 k\end{array}\right)$ and Lemma 19 applied with $b=k+|i| \geq 2 k, n=|j|-|i|$ insures that $|j|-|i|=i-j$ divides $\int c m(2 k)\left(P_{2 k}(j)-P_{2 k}(i)\right)$. 
2. Case $n=2 k$ and $j \in]-\infty,-k]$ and $i \in]-k, k]$. Then $P_{2 k}(i)-P_{2 k}(j)=$ $-\left(\begin{array}{c}k+|j| \\ 2 k\end{array}\right)$. Let $n^{\prime}=k+|j|, k^{\prime}=2 k$ and $p^{\prime}=i-j=i+|j|$. Then $0 \leq$ $n^{\prime}-k^{\prime}<p^{\prime} \leq n^{\prime}$, and Lemma 17 insures that $i-j$ divides $\operatorname{lcm}\left(k^{\prime}\right)\left(\begin{array}{c}n^{\prime} \\ k^{\prime}\end{array}\right)=$ $l c m(2 k)\left(P_{2 k}(j)-P_{2 k}(i)\right)$.

3. Case $n=2 k$ and $j \in]-\infty,-k]$ and $i \in] k,+\infty\left[\right.$. Then $P_{2 k}(i)-P_{2 k}(j)=$ $\left(\begin{array}{c}k+i-1 \\ 2 k\end{array}\right)-\left(\begin{array}{c}k+|j| \\ 2 k\end{array}\right)$.

- subcase $|j| \leq i-1$ Let $n^{\prime}=i$ and $i^{\prime}=|j|$. As $i^{\prime} \leq n^{\prime}-1$ Lemma 20 (5) applies and insures that $n^{\prime}+i^{\prime}=i+|j|=i-j$ divides $l c m(2 k) B\left(n^{\prime}, k, i^{\prime}\right)=$ $l c m(2 k)\left(P_{2 k}(i)-P_{2 k}(j)\right)$.

- subcase $|j| \geq i$ Let $n^{\prime}=|j|+1$ and $i^{\prime}=i-1$. Again by Lemma 20 (5), $n^{\prime}+i^{\prime}=i+|j|=i-j$ divides $l c m(2 k) B\left(n^{\prime}, k, i^{\prime}\right)=l c m(2 k)\left(P_{2 k}(j)-P_{2 k}(i)\right)$.

4. Case $n=2 k$ and $i, j \in]-k,-k]$. Clear as $P_{2 k}(i)=P_{2 k}(j)=0$.

5. Case $n=2 k$ and $j \in]-k,-k]$ and $i \in] k,+\infty\left[\right.$. Then $P_{2 k}(i)-P_{2 k}(j)=$ $\left(\begin{array}{c}k+i \\ 2 k\end{array}\right)$. Let $n^{\prime}=k+i, k^{\prime}=2 k$ and $p^{\prime}=i-j$. We have $0 \leq n^{\prime}-k^{\prime}<p^{\prime} \leq n^{\prime}$, hence by Lemma 17, $p^{\prime}=i-j$ divides $l c m(2 k)\left(\begin{array}{c}k+i \\ 2 k\end{array}\right)$.

6. Case $n=2 k$ and $i, j \in] k,+\infty\left[\right.$. Then $P_{2 k}(i)-P_{2 k}(j)=\left(\begin{array}{c}k+i-1 \\ 2 k\end{array}\right)-$ $\left(\begin{array}{c}k+j-1 \\ 2 k\end{array}\right)$ with $2 k \leq k+j-1$, we can thus conclude using Lemma 19

7. Case $n=2 k+1$ and $i, j \in]-\infty,-k\left[\right.$. Then $P_{2 k+1}(i)-P_{2 k+1}(j)=-\left(\begin{array}{c}k+|i| \\ 2 k+1\end{array}\right)+$ $\left(\begin{array}{l}k+|j| \\ 2 k+1\end{array}\right)$ : applying Lemma 19 with $b=|i|, n=|j|-|i|$ we conclude that $n=i-j$ divides $l c m(2 k+1)\left(P_{2 k+1}(i)-P_{2 k+1}(j)\right)$.

8. Case $n=2 k+1$ and $j \in]-\infty,-k\left[\right.$ and $i \in[-k, k]$. Then $P_{2 k+1}(i)-P_{2 k+1}(j)=$ $\left(\begin{array}{c}k+|j| \\ 2 k+1\end{array}\right)$. We conclude as in case 2. above, with Lemma 17

9. Case $n=2 k+1$ and $j \in]-\infty,-k[$ and $i \in] k,+\infty\left[\right.$. Then $P_{2 k+1}(i)-P_{2 k+1}(j)=$ $\left(\begin{array}{c}k+i \\ 2 k+1\end{array}\right)+\left(\begin{array}{c}k+|j| \\ 2 k+1\end{array}\right)$. - subcase $|j| \leq i-1$ : let $n^{\prime}=i, i^{\prime}=|j|$ and apply Lemma 20 (6).

- subcase $i \leq|j|-1$ : let $n^{\prime}=|j|, i^{\prime}=i$ and apply Lemma 20 (6).

- subcase $i=|j|$ : then $P_{2 k+1}(i)-P_{2 k+1}(j)=2\left(\begin{array}{c}k+i \\ 2 k+1\end{array}\right)$; Lemma 17 applied with $n^{\prime}=k+i, k^{\prime}=2 k+1$ and $p^{\prime}=i\left(0 \leq n^{\prime}-k^{\prime}<p^{\prime} \leq n^{\prime}\right.$ hold $)$, implies that $i$ divides $l c m(2 k+1)\left(\begin{array}{c}k+i \\ 2 k+1\end{array}\right)$, hence $2 i=i-|j|$ divides $l c m(2 k+1)\left(P_{2 k+1}(i)-\right.$ $\left.P_{2 k+1}(j)\right)$.

10. Case $n=2 k+1$ and $i, j \in[-k,-k]$. Trivial since then $P_{2 k+1}(i)=P_{2 k+1}(j)=$ 0 .

11. Case $n=2 k+1$ and $j \in[-k, k]$ and $i \in] k,+\infty\left[\right.$. Then $P_{2 k+1}(i)-P_{2 k+1}(j)=$ $\left(\begin{array}{c}k+i \\ 2 k+1\end{array}\right)$. Let $n^{\prime}=k+i, k^{\prime}=2 k+1$, and $p^{\prime}=i-j$ : as $0 \leq n^{\prime}-k^{\prime}=i-k-1$, 
as $|j| \leq k$ and $i>k, i-k-1<p^{\prime}=i-j \leq n^{\prime}$, the hypothesis of Lemma 17 hold and Lemma 17 yields $i-j$ divides $l c m(2 k+1)\left(P_{2 k+1}(i)-P_{2 k+1}(j)\right)$.

12. Case $n=2 k+1$ and $i, j \in] k,+\infty[$. Similar to Case 7 .

\section{Non polynomial functions having integral difference ratio}

Let us mention a straightforward consequence of Theorem 16 .

Corollary 21. There are non polynomial functions $\mathbb{Z} \rightarrow \mathbb{Z}$ having integral difference ratio.

Proof. In fact there are uncountably many such functions: let $a_{n}$ be any element of $l c m(n) \mathbb{N}, n \in \mathbb{N}$, then $\sum_{n \in \mathbb{N}} a_{n} P_{n}(x)$ has integral difference ratio.

We now explicit some non polynomial functions having integral difference ratio. We first briefly recall such examples $\mathbb{N} \rightarrow \mathbb{Z}$ (Theorem 23) obtained in [3] and then explicit a function $\mathbb{Z} \rightarrow \mathbb{Z}$ (Theorem 26).

Lemma 22. For all $k$, we have $\operatorname{lcm}(k)$ divides $\frac{(2 k) !}{k !}$.

Proof. We have $\operatorname{lcm}(2 k)=\prod_{p \text { prime }} p^{N(p)}$ with $N(p)=\sup \left\{i \mid p^{i} \leq 2 k\right\}$. For $p$ prime, let $M(p)$ be the largest integer divided by $p^{N(p)}$ and $\leq 2 k$. Then $2 M(p)>2 k$ hence $M(p)>k$. In particular, $M(p)$ hence $p^{N(p)}$ divides $(2 k) ! / k !$. As a product of pairwise coprime integers, $\operatorname{lcm}(2 k)=\prod_{p \text { prime }} p^{N(p)}$ also divides $(2 k) ! / k !$.

Theorem 23. Let e be the Neper constant. The following functions $\mathbb{N} \rightarrow \mathbb{Z}$ have integral difference ratio:

$$
f: x \mapsto\left\{\begin{array}{ll}
1 & \text { if } x=0 \\
\lfloor\text { e } x !\rfloor & \text { if } x \in \mathbb{N} \backslash\{0\}
\end{array} \quad f_{h}: x \mapsto\left\{\begin{array}{l}
\lfloor\sinh (1) x !\rfloor \text { if } x \text { odd } \\
\lfloor\cosh (1) x !\rfloor \text { if } x \text { even }
\end{array}\right.\right.
$$

Remark 24. Function $\lfloor e x$ ! 」 does not have integral difference ratio (cf. [3]).

Proof. Recall Taylor-Lagrange formula applied to the real function $t \mapsto e^{t}$ : for all $t \in \mathbb{R}$,

$$
e^{t}=\left(\frac{1}{0 !}+\frac{t}{1 !}+\frac{t^{2}}{2 !}+\cdots+\frac{t^{k-1}}{(k-1) !}+\frac{t^{k}}{k !}\right)+e^{\theta t} \frac{t^{k+1}}{(k+1) !}
$$

for some $0<\theta<1$ depending on $k$ and $t$.

Let $f: \mathbb{N} \rightarrow \mathbb{Z}$ be the function associated with the Newton series $f(x)=$ $\sum_{n \in \mathbb{N}} n !\left(\begin{array}{l}x \\ n\end{array}\right)$. Theorem 12 insures that $f$ has integral difference ratio. By (7) 
above, there exists $\theta$, with $0<\theta<1$ such that

$$
\begin{aligned}
f(x) & =\sum_{n \in \mathbb{N}} n !\left(\begin{array}{l}
x \\
n
\end{array}\right)=\sum_{n=0}^{x} \frac{x !}{(x-n) !}=x !\left(\frac{1}{x !}+\frac{1}{(x-1) !}+\cdots+\frac{1}{1 !}+\frac{1}{0 !}\right) \\
& =x !\left(e-e^{\theta} \frac{1}{(x+1) !}\right)
\end{aligned}
$$

Thus, $e x !=f_{a}(x)+\frac{e^{\theta}}{(x+1)}$ For $x \in \mathbb{N}, x \geq 2$, we have $0<e^{\theta} /(x+1)<e / 3<1$ and the last equality yields $f(x)=\lfloor e x !\rfloor$. Also, $f(0)=1<2=\left\lfloor e 0\right.$ ! 」, $f_{1}(1)=$ $2=\lfloor e 1 !\rfloor$.

Similarly, Lemma 22 and Theorem 12 insure that $f_{h}(x)=\sum_{n \in \mathbb{N}}(2 n) !\left(\begin{array}{c}x \\ 2 n\end{array}\right)$ has integral difference ratio and a similar computation yields

$f_{h}(x)=\sum_{k \in \mathbb{N}}(2 k) !\left(\begin{array}{c}x \\ 2 k\end{array}\right)=\sum_{k=0}^{\lfloor x / 2\rfloor} \frac{x !}{(x-2 k) !}= \begin{cases}x ! \sum_{k=0}^{\frac{x-1}{2}} \frac{1}{(2 k+1) !} & \text { if } x \text { odd } \\ x ! \sum_{k=0}^{\frac{x}{2}} \frac{1}{(2 k) !} & \text { if } x \text { even }\end{cases}$

Applying Taylor-Lagrange formula, we get $\theta_{o}, \theta_{e}$ in $] 0,1\left[\right.$ such that $f_{h}(x)=$ $x !\left(\sinh (1)-\frac{\sinh \left(\theta_{o}\right)}{(x+1) !}\right)$ if $x$ is odd and $f_{h}(x)=x !\left(\cosh (1)-\frac{\sinh \left(\theta_{e}\right)}{(x+1) !}\right)$ if $x$ is even. Whence the result as in the previous case.

It is easy to lift the integral difference ratio property from functions $\mathbb{N} \rightarrow \mathbb{Z}$ to functions $\mathbb{Z} \rightarrow \mathbb{Z}$.

Proposition 25. Suppose $f: \mathbb{N} \rightarrow \mathbb{Z}$ has integral difference ratio and let $g$ : $\mathbb{Z} \rightarrow \mathbb{Z}$ be such that $g(x)=f\left(x^{2}\right)$. Then $g$ has integral difference ratio. In particular, there is a function $g: \mathbb{Z} \rightarrow \mathbb{Z}$ having integral difference ratio and such that $g(x) \in\left\{\left\lfloor e\left(x^{2}\right) !\right\rfloor,\left\lfloor e\left(x^{2}\right) !\right\rfloor-1\right\}$.

Proof. Since $a^{2}-b^{2}=\operatorname{divides} f\left(a^{2}\right)-f\left(b^{2}\right)=g(a)-g(b)$ so does $a-b$.

Here is an example of a non polynomial function $\mathbb{Z} \rightarrow \mathbb{Z}$ having integral difference ratio and which is not relevant to Proposition 25.

Theorem 26. The function defined by $n \mapsto \sqrt{\frac{e}{\pi}} \times \frac{\Gamma(1 / 2)}{2 \times 4^{n} \times n !} \int_{1}^{\infty} e^{-t / 2}\left(t^{2}-\right.$ $1)^{n} d t$ for $n \geq 0$ and by $f(n)=-f(|n|-1)$ for $n<0$ maps $\mathbb{Z}$ into $\mathbb{Z}$ and has integral difference ratio.

Proof. Let $f: \mathbb{Z} \rightarrow \mathbb{Z}$ have $\mathbb{Z}$-Newton expansion $f(x)=\sum_{k \in \mathbb{N}} \frac{2 k !}{k !} P_{2 k}(x)$, i.e. $a_{2 k}=(2 k) ! / k$ ! and $a_{2 k+1}=0$. It is clearly nonpolynomial and, by Theorem 16 . it has integral difference ratio.

For $n \geq 0$ we have, by [8], page 2, formula 0.126 , and page 917 formulas 8.432 $1 \& 3$, 


$$
\begin{aligned}
f(n) & =\sum_{k=0}^{n} \frac{2 k !}{k !} \frac{(n+k)(n+k-1) \cdots(n-k+2)(n-k+1)}{(2 k) !}=\sum_{k=0}^{n} \frac{(n+k) !}{k !(n-k) !} \\
& =\sqrt{\frac{e}{\pi}} \times K_{n+\frac{1}{2}}\left(\frac{1}{2}\right)=\sqrt{\frac{e}{\pi}} \times \frac{\Gamma\left(\frac{1}{2}\right)}{2 \times 4^{n} \times n !} \int_{1}^{\infty} e^{-\frac{t}{2}}\left(t^{2}-1\right)^{n} d t \\
f(-n) & =\sum_{k=0}^{n} \frac{2 k !}{k !} \frac{(-n+k)(-n+k-1) \cdots(-n-k+2)(-n-k+1)}{(2 k) !} \\
& =\sum_{k=0}^{n}(-1)^{2 k} \frac{(n+k-1) \cdots(n-k)}{k !}=\sum_{k=0}^{n} \frac{(n+k-1) !}{k !(n-k-1) !}=f(n-1)
\end{aligned}
$$

where $K_{\nu}(x)=\int_{0}^{\infty} e^{-x \cosh t} \cosh (\nu t) d t$ is associated with the Bessel function of the third kind.

\section{Conclusion}

We here studied functions having the integral difference ratio property; these functions appeared in two ways at least:

(i) as the functions such that lattices of regular subsets of $\mathbb{N}$ are closed under $f^{-1}$ (see [2]), and [10).

(ii) as the functions uniformly continuous in a variety of finite groups (see

We characterized the class of integral difference ratio functions from $\mathbb{Z}$ (resp. $\mathbb{N}$ ) to $\mathbb{Z}$ via their Newton series expansions on bases of polynomials with rational coefficients. This enabled us to exhibit non polynomial such functions.

Integral difference ratio functions can be seen as the solution for algebra $\mathbb{Z}$ of a general problem: which functions preserve a family of congruences on a given structure? Functions preserving all congruences on an algebra have been studied in universal algebra; it was known [11 that there exist such functions from $\mathbb{Z}$ to $\mathbb{Z}$ which are polynomials with non-integer coefficients. Our contribution to the study of congruence preserving functions on $\mathbb{Z}$ is (i) to characterize the congruence preserving functions from $\mathbb{Z}$ to $\mathbb{Z}$ as the integral difference ratio functions and (ii) to give an example of a non polynomial Bessel like congruence preserving function.

\section{References}

1. M. Benois, Parties Rationnelles du Groupe Libre, C. R. Acad. Sci. Paris Série A, 269, pp 1188-1190, 1969.

2. P. CÉGielski and S. GrigoriefF And I. Guessarian, On Lattices of Regular Sets of Natural Integers Closed under Decrementation, Information Processing Letters 114(4):197-202, 2014. Preliminary version on arXiv, 2013. 
3. P. Cegielski, S. Grigorieff, I. Guessarian, Newton expansion of functions over natural integers having integral difference ratios. To be published in Int. J. Number Theory. Preliminary version on arXiv, 2013.

4. P. Dusart, Estimates of some functions over primes without Riemann hypothesis, unpublished. Preprint version on arXiv, 2010.

5. B. FARHI, Nontrivial lower bounds for the least common multiple of some finite sequences of integers, Journal of Number Theory, 125:393-411, 2007.

6. B. FARHi And D. Kane, New Results on the Least Common Multiple of Consecutive Integers, Proceedings of the AMS, 137(6):1933-1939, 2009.

7. Andrew Granville, Binomial coefficients modulo prime powers, Conference Proceedings of the Canadian Mathematical Society, 20:253-275, 1997.

8. I. S. Grashteyn, I. M. Ryzhik, Table of Integrals, Series, and Products, 7th edition, Academic Press, 2007.

9. S. Hong, G. QIAN AND Q. TAN, The least common multiple of a sequence of products of linear polynomials, Acta Mathematica Hungarica, 135(1-2):160-167, 2011.

10. J.-É. PIN AND P.V. Silva, On profinite uniform structures defined by varieties of finite monoids, International Journal of Algebra and Computation, 21:295-314, 2011.

11. A.F. PiXley, Functional and affine completeness and arithmetical varieties, Algebras and Orders, J. Rosenberg and G. Sabidussi (eds.), Kluwer Acad. Pub., 317-357, 1993.

12. Guoyou Qian And Shaofang Hong, Asymptotic behavior of the least common multiple of consecutive arithmetic progression terms, Archiv der Mathematik, 100(4):337-345, 2013. 